\title{
CERTAIN $n$th ORDER DIFFERENTIAL INEQUALITIES IN THE COMPLEX PLANE
}

\author{
BY
}

H. S. AL-AMIRI

\begin{abstract}
Let $w(z)$ be regular in the unit disc $U:|z|<1$, with $w(0)=0$ and let $h(r, s, t)$ be a complex function defined in a domain $D$ of $C^{3}$. The author determines conditions on $h$ such that if

$$
\left|h\left(D^{n} w(z), D^{n+1} w(z),(n+2)\left(D^{n+2} w(z)-D^{n+1} w(z)\right)\right)\right|<1
$$

$z \in U$, then $|w(z)|<1$ for $z \in U$ and $n=0,1,2, \ldots$ Here $D^{n} w(z)=$ $\left(z /(1-z)^{n+1} * w(z)\right.$, where $*$ stands for the Hadamard product (convolution). Some applications of the results to certain differential equations are given.
\end{abstract}

1. Introduction. Let $A$ be the family of functions $w(z)$ regular in the unit disc $U:|z|<1$, with $w(0)=0$. For $w(z) \in A$, let

$$
D^{n} w(z)=z\left(z^{n-1} w(z)\right)^{(n)} / n !
$$

where $n \in N_{0}=0,1,2, \ldots$ and $z \in U$.

Ruscheweyh [4] observed that (1) can also be written in the form

$$
D^{n} w(z)=\left(z /(1-z)^{n+1}\right) * w(z),
$$

where $(*)$ stands for the Hadamard product, that is, if

$$
f(z)=\sum_{1}^{\infty} a_{n} z^{n}, \quad g(z)=\sum_{1}^{\infty} b_{n} z^{n}, \quad \text { then } \quad f(z) * g(z)=\sum_{1}^{\infty} a_{n} b_{n} z^{n} .
$$

Recently, Miller [2], and Miller and Mocanu [3] have determined the complex valued functions $h(r, s, t)$ defined in a domain of $C^{3}$ such that if $w(z) \in A$ satisfies the inequalities

$$
\left|h\left(w(z), z w^{\prime}(z), z^{2} w^{\prime \prime}(z)\right)\right|<1 \text { for } z \in U, \text { then }|w(z)|<1 \text { for } z \in U \text {. }
$$

The purpose of this note is to extend and to generalize the above results. To be specific we shall determine the class of complex valued functions $H_{n}$ defined in some domain $D$ of $C^{3}$ such that for $w(z) \in A$ and $h \in H_{n}$ satisfying

$$
\left|h\left(D^{n} w(z), D^{n+1} w(z),(n+2)\left(D^{n+2} w(z)-D^{n+1} w(z)\right)\right)\right|<1
$$

for $z \in U$ and $n \in N_{0}$, then $|w(z)|<1$ for $z \in U$.

Received by the editors August 16, 1977 and, in revised form, January 5, 1978. 
Applications of this result in the theory of differential equations are also given.

2. Results and applications. We need the following lemma of Miller and Mocanu [3].

Lemma. Let $w(z) \in A$ with $w(z) \not \equiv 0$ in $U$. If $z_{0}=r_{0} e^{i \theta_{0}}, 0<r_{0}<1$ and $\left|w\left(z_{0}\right)\right|=\max _{|z| \leq r_{0}}|w(z)|$, then

(i) $z_{0} w^{\prime}\left(z_{0}\right) / w\left(z_{0}\right)=m$, and

(ii) $\operatorname{Re}\left\{z_{0} w^{\prime \prime}\left(z_{0}\right) / w^{\prime}\left(z_{0}\right)+1\right\} \geq m$,

where $m \geq 1$.

Part (i) of the Lemma can also be found in [1].

Definition. For $n \in N_{0}$, let $H_{n}$ be the set of functions $h(r, s, t): C^{3} \rightarrow C$ such that

(i) $h(r, s, t)$ is continuous in a domain $D$ of $C^{3}$,

(ii) $(0,0,0) \in D$ and $|h(0,0,0)|<1$,

(iii)

when

$$
\left|h\left(e^{i \theta}, \frac{n+m}{n+1} e^{i \theta}, \frac{n(m-1) e^{i \theta}+L}{n+1}\right)\right|>1
$$

$$
\left(e^{i \theta}, \frac{n+m}{n+1} e^{i \theta}, \frac{n(m-1) e^{i \theta}+L}{n+1}\right) \in D
$$

where $\operatorname{Re} e^{-i \theta} L \geq m(m-1)$ for $\theta$ and real $m \geq 1$.

We now state and prove our main result.

THEOREM 1. Let $w \in A$ and $n \in N_{0}$ satisfying:

(i) $\left(D^{n} w(z), D^{n+1} w(z),(n+2)\left(D^{n+2} w(z)-D^{n+1} w(z)\right)\right) \in D$,

(ii) $\left|h\left(D^{n} w(z), D^{n+1} w(z),(n+2)\left(D^{n+2} w(z)-D^{n+1} w(z)\right)\right)\right|<1$, when $z \in U$. Then $\left|D^{n} w(z)\right|<1$ for $z \in U$.

Proof. Using (2) we have

$$
z\left(D^{k} w(z)\right)^{\prime}=(k+1) D^{k+1} w(z)-k D^{k} w(z), \quad k \in N_{0} .
$$

Let

$$
\ell(z)=D^{n} w(z)
$$

From (3) and (4) with $k=n$ we obtain

$$
(n+1) D^{n+1} w(z)=n \ell(z)+z \ell^{\prime}(z) .
$$

Differentiation of (5) and using (3) with $k=n+1$ lead to

(6) $(n+2)\left(D^{n+2} w(z)-D^{n+1} w(z)\right)=\left(-n \ell(z)+n z \ell^{\prime}(z)+z^{2} \ell^{\prime \prime}(z)\right) /(n+1)$. 
Now suppose there exists $z_{0}=r_{0} e^{i{ }_{0}}, 0<r_{0}<1$ such that

$$
\left|D^{n} w\left(z_{0}\right)\right|=\max _{|z| \leq r_{0}}\left|D^{n} w(z)\right|
$$

Since $D^{n} w(0)=0$, the proof of Theorem 1 would be completed if (7) is shown to be impossible for any $z_{0} \in U$. Applying the Lemma to (4), (5) and (7) yield

$$
\begin{aligned}
& D^{n} w\left(z_{0}\right)=\ell\left(z_{0}\right)=e^{i \theta}, \\
& D^{n+1} w\left(z_{0}\right)=\frac{m+n}{n+1} e^{i \theta},
\end{aligned}
$$

and

$$
\operatorname{Re}\left\{z_{0}^{2} \ell^{\prime \prime}\left(z_{0}\right) / z_{0} \ell^{\prime}\left(z_{0}\right)+1\right\}=\operatorname{Re} z_{0}^{2} \ell^{\prime \prime}\left(z_{0}\right) \cdot e^{-i \theta} \geq m(m-1)
$$

Thus substitution of (8), (9) and (10) in (6) give

$$
(n+2)\left(D^{n+2} w\left(z_{0}\right)-D^{n+1} w\left(z_{0}\right)\right)=\left(n(m-1) e^{i \theta}+L\right) /(n+1),
$$

where $L=z_{0}^{2} \ell^{\prime \prime}\left(z_{0}\right)$ and therefore $\operatorname{Re} \operatorname{Le}^{-i \theta} \geq m(m-1), m \geq 1$.

Finally from (8), (9), (11) and part (iii) of the Definition assuming part (i) of the hypothesis we get

$$
\begin{gathered}
\left|h\left(D^{n} w\left(z_{0}\right), D^{n+1} w\left(z_{0}\right),(n+2)\left(D^{n+2} w\left(z_{0}\right)-D^{n+1} w\left(z_{0}\right)\right)\right)\right| \\
=\left|h\left(e^{i \theta},\left(\frac{m+n}{n+1}\right) e^{i \theta}, \frac{n(m-1) e^{i \theta}+L}{n+1}\right)\right|>1,
\end{gathered}
$$

where $\operatorname{Re} \mathrm{Le}^{-i \theta} \geq m(m-1)$. This contradicts part (ii) of the hypothesis. Thus the proof is completed.

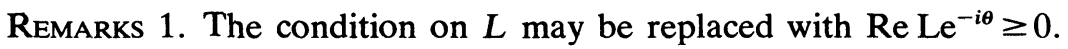

2. For $n=0$, Theorem 1 reduces to Theorem 2 of [3] for the class, $A$, and also includes Theorem 2 of [2].

Let $h_{0}(r, s, t)=s$. It is obvious that $h_{0} \in H_{n}$. By iteration of Theorem 1 applied to $h_{0}$ we get the following corollary.

Corollary 1. ||$D^{n+1} w(z)|<1 \Rightarrow| w(z) \mid<1$ for $z \in U$ and $n \in N_{0}$. Theorem 1 and Corollary 1 give the following.

Corollary 2. If $h(r, s, t)$ and $w(z)$ are as in Theorem 1 , then $|w(z)|<1$ for $z \in U$.

Corollary 2 can be used to show that certain $n$th order differential equations have bounded solutions. 
Theorem 2. Let $h(r, s, t)$ and $w(z)$ be as in Theorem 1 and let $b(z)$ be a regular function in $U$ with $|b(z)|<1, z \in U$. If the differential equation

$$
h\left(D^{n} w(z), D^{n+1} w(z),(n+2)\left(D^{n+2} w(z)-D^{n+1} w(z)\right)\right)=b(z)
$$

has a solution $w(z)$ regular in the unit disc $U$ then $|w(z)|<1$.

We close this brief note with some examples and applications, see [2] and [3] for further details.

EXAMPLES. It is easy to check that each of the following functions are in $H_{n}$.

$$
\begin{array}{ll}
h_{1}(r, s, t)=\alpha r+s, & \operatorname{Re} \alpha \geq 0, D=C^{3}, n \in N_{0} . \\
h_{2}(r, s, t)=2 s /(1-r), & D=(C-\{1\}) \times C^{2}, n \in N_{0} . \\
h_{3}(r, s, t)=r^{i} s^{j}\left(t^{k}+r^{\ell}\right), & D=C^{3}, i, j, k \in N_{0} \quad \text { and } \quad \ell \in N . \\
h_{4}(r, s, t)=r+s+t, & D=C^{3}, n \in N_{0} . \\
h_{5}(r, s, t)=-r+2 s+2 t, & D=C^{3}, n \in N .
\end{array}
$$

Applying Corollary 2 to $h_{4}$ with $n=0$ we obtain the Euler equation

$$
z^{2} w^{\prime \prime}(z)+z w^{\prime}(z)+w(z)=b(z) .
$$

If $|b(z)|<1$ then we must have $|w(z)|<1$.

Similarly applying the same Corollary to $h_{5}$ with $n=1$ we get

$$
z^{3} w^{\prime \prime \prime}(z)+4 z^{2} w^{\prime \prime}(z)+z w^{\prime}(z)=b(z) .
$$

And if $|b(z)|<1$ and the above differential equation has a solution $w(z)$ regular in $U$ then $|w(z)|<1$ for $z \in U$.

If we apply same corollary to $h_{3}, r=j=k=\ell=1, n=2$, then the resulting nonlinear differential equation

$$
z w^{\prime}\left(2 z w^{\prime}+z^{2} w^{\prime \prime}\right)\left(2 z w^{\prime}+3 z^{2} w^{\prime \prime}+z^{3} w^{\prime \prime \prime}\right)=4 b(z)
$$

would have a bounded solution $|w(z)|<1$ for $z \in U$ provided that there exists a solution $w(z)$ regular in the unit disc $U$ when $|b(z)|<1$.

Problem. A natural problem arises from the results and examples of this note; namely does there exist a function $h\left(r_{1}, r_{2}, \ldots, r_{n}\right)$ defined in some suitable domain $D$ of $C^{n}$ such that if

$$
\left|h\left(w(z), z w^{\prime}(z), \ldots, z^{n} w^{(n)}(z)\right)\right|<1 \quad \text { for } \quad z \in U,
$$

then $|w(z)|<1$ for $z \in U$.

\section{REFERENCES}

1. I. S. Jack, Functions starlike and convex of order $\alpha$, J. London Math. Soc. (2) 3 (1971), 469-474. 
2. S. S. Miller, A class of differential inequalities implying boundedness, III. J. of Math. 20 (1976), 647-649.

3. S. S. Miller and P. T. Mocanu, Second order differential inequalities in the complex plane, to appear.

4. S. Ruscheweyh, New criteria for univalent functions, Proc. Amer. Math. Soc. vol. 49 (1975), 109-115.

Department of Mathematics

Bowling Green State University

Bowling Green, OHio 43403 